\title{
Array configuration studies for the SKA - Implementation of figures of merit based on SDR
}

\author{
Dharam V. Lal*i \\ Max-Planck-Institut für Radioastronomie \\ Auf dem Hügel 69, 53121 Bonn, Germany \\ E-mail: dharamempifr-bonn.mpg.de \\ Andrei P. Lobanov \\ Max-Planck-Institut für Radioastronomie \\ Auf dem Hügel 69, 53121 Bonn, Germany \\ E-mail: alobanov@mpifr-bonn.mpg.de
}

\begin{abstract}
The Square Kilometre Array is going to become operational at the time when several new large optical, X-ray and Gamma-ray telescopes are expected to be working. The main drive for building the SKA is a significant improvement of sensitivity that would widen the general scope of the centimetre-wavelength radio science. To this end, a thorough design studies should be made, in order to ensure that the SKA becomes a competitive counterpart of the facilities at other wavebands.

To quantify imaging performance of the SKA configurations, we are implementing figures of merit based on spatial dynamic range (SDR). This work is focused on extensive numerical tests of the analytical, SDR-based figures of merit derived in the SKA Memo 38 (A. Lobanov). Here, we present our preliminary results.
\end{abstract}

From Planets to Dark Energy: the Modern Radio Universe

October 1-5 2007

The University of Manchester, $U K$

\footnotetext{
*Speaker.

$\dagger$ This effort/activity is supported by the European Community Framework Programme 6, Square Kilometer Array Design Studies (SKADS), contract no 011938. DVL thanks Maxim A. Voronkov for helping with the simulator in aips ++ and the related scripts, and Borkowski, K. \& Fukushima, T. for sharing their "Cartesian to geodetic coordinates transform" subroutines.
} 


\section{Key Terminologies}

1. Spatial Dynamic Range The ratio of the largest adequately imaged structure and the synthesized beam. It depends on the correlator integration time, channel width, and $(u, v)$-plane coverage.

2. Figures of Merit Any parameter which is a measure of $(u, v)$-plane coverage; e.g., spatial dynamic range (SDR), RMS noise levels, synthesized beam size, etc.

3. $u v$-gap parameter or $\Delta u / u$ A measure of the quality of the $(u, v)$-plane coverage, characterising the relative size of "holes" in the Fourier plane. In the simplest case of a circular $(u, v)$-plane coverage, the $u v$-gap parameter,

$$
\frac{\Delta u}{u}=\frac{\left(u_{2}-u_{1}\right)}{u_{2}},
$$

where $u_{1}, u_{2}\left(u_{2}>u_{1}\right)$ are the $u v$-radii of two adjacent baselines.

\section{Description of the Work Methodology}

1. We use the simulator in aips ++ (ver 1.9 (build 1556)) package to generate a visibility data set for an input array configuration to determine and study the figures of merit for quantifying the properties of $(u, v)$-plane coverage and its effect on imaging.

2. Next, we vary the array configuration, generate a new visibility data set, and repeat this exercise many times to complete the full parameter space of SDR.

3. Several scripts, both in Fortran and glish languages, have been written to accomplish our goal. We also make use of "classic AIPS" for our imaging analysis.

\section{Robustness Checks}

The array can be placed at any position on the Earth's surface, but since, we understand the behaviour of the baseline vector for the array placed at the Earth's pole, when a source being observed is at 90 deg declination, we use this case to understand the robustness of the output from the aips ++ simulator.

We generate spiral arrays in many different ways. We use these array configurations and perform identical pipeline analysis to conduct robustness checks.

\section{Determination of the "Figures of Merit"}

Experiment 1 We use equiangular (logarithmic) spiral array configuration, consisting of a station at the origin, three spiral arms and five stations in each arm, as the geometry. The range of baselines used are between 20 to $100 \mathrm{~m}$ and 20 to $5000 \mathrm{~m}$.

We make dirty maps (with each image being 4096 pixels $\times 4096$ pixels and every pixel being 2 arcsec) for each simulated visibility data set, and use these maps to determine map characteristics, such as, peak surface brightness, noise level and beam area. We use these results to study the figures of merit for quantifying the properties of $(u, v)$-plane coverage. 
Experiment 2 Next, in order to probe finer $u v$-gap parameters, we next perform simulations keeping largest baseline length, $\mathrm{B}_{\max }(=5 \mathrm{~km})$ constant and instead vary $N$; 1.e., we populate a large number of antennas on to a single equiangular spiral arm pattern in a logarithmic fashion In addition, as we add more number of stations for a new array configuration, we increase the integration time, thereby, we keep identical sensitivities for each new visibility data set.

Thus, we generate a range of visibility data sets to probe $u v$-gap parameter from $0.45(N=50)$ to $0.01(N=640)$. We make dirty and CLEAN maps (with each image being 8192 pixels $\times 8192$ pixels and every pixel being 3 arcsec) for each simulated visibility data set, and use the CLEAN maps to determine several map characteristics.

Simulations: Parameters Used We use the following parameters for the generation of a range of visibility data sets:

\begin{tabular}{|c|c|c|}
\hline \multicolumn{2}{|c|}{ Telescope settings } & Input group of source components \\
\hline Frequency & : $\quad$ L band $(1.4 \mathrm{GHz})$ & Experiment 1 \\
\hline Antenna & : $25 \mathrm{~m}(\mathrm{VLA})$ & (Constant $N$ and Varying $\mathrm{B}_{\max }$ ) \\
\hline Bandwidth & : $\quad 3.2 \mathrm{MHz}$ & Six Gaussian components; \\
\hline No. of channels & $: \quad 1$ & typical source size, $\sim 1^{\prime}$ \\
\hline Direction (J2000) & $: \quad 00: 00: 00+90.00 .00$ & \\
\hline Elevation limit & : $12 \mathrm{deg}$ & Experiment 2 \\
\hline Shadow limit & : 0.001 & $\left(\right.$ Constant $\mathrm{B}_{\max }$ and Varying $\left.N\right)$ \\
\hline Start_time (IAT) & $: \quad 22 / 08 / 2007 / 06: 00$ & Seven Gaussian components; \\
\hline Stop_time (IAT) & $: \quad$ 22/08/2007 / 18:00 & source size range: $0.1^{\prime} \times 0.1^{\prime}-120^{\prime} \times 40^{\prime}$ \\
\hline
\end{tabular}

\section{Preliminary Inferences}

$\Rightarrow$ The behaviour of figures of merit and hence the SDR does not seem to have a simple dependence on $\Delta u / u$. Our results show that close to small $u v$-gap parameter values, the (nearly) linear relationship does not hold good.

$\Rightarrow$ Our simulations show that the $u v$-gap parameter can be used to relate the $(u, v)$-plane coverage to the characteristics of the map. Although, we cover a small part of the full parameter space to be probed, the preliminary study demonstrates a valuable result which is important to put constraints on the SKA design studies.

\section{Conclusions}

To make the SKA a competitive instrument that would match the capabilities of future optical and X-ray telescopes, two basic conditions must be fullfilled: (i) resolution $\lesssim 1$ mas at the high end of frequency and (ii) Fourier plane filling factor $\Delta u / u \lesssim 0.05$ over entire range of $(u, v)$-plane coverage.

Any compromise in either of these two conditions would reduce the imaging capability and narrow the scientific scope of the SKA. 